\title{
High-extraction-efficiency, nanosecond bidirectional ring amplifier with twin pulses
}

\author{
Tiancheng $\mathrm{Yu}^{1,2, \dagger}$, Jiangtao Guo ${ }^{3,4, \dagger}$, Gang $\mathrm{Xia}^{3,4}$, Xiang Zhang ${ }^{1,2}$, Fan Gao ${ }^{1,2}$, Jiangfeng Wang ${ }^{3}$, \\ Wei Fan ${ }^{3}$, and Xiao Yuan ${ }^{1,2}$ \\ ${ }^{1}$ School of Optoelectronic Science and Engineering \& Collaborative Innovation Center of Suzhou Nano Science and Technology, \\ Soochow University, Suzhou 215006, China \\ ${ }^{2}$ Key Laboratory of Advanced Optical Manufacturing Technologies of Jiangsu Province and Key Laboratory of Modern Optical \\ Technologies of Ministry of Education, Suzhou 215006, China \\ ${ }^{3}$ Shanghai Institute of Optics and Fine Mechanics, Chinese Academy of Sciences, Shanghai 201800, China \\ ${ }^{4}$ University of Chinese Academy of Sciences, Beijing 100049, China \\ (Received 27 November 2018; revised 9 January 2019; accepted 12 March 2019)
}

\begin{abstract}
The output performances of a bidirectional ring amplifier with twin pulses are demonstrated. Compared to the extraction efficiency of $32 \%$ for single-pulse injection, the extraction efficiency of stored energy for twin-pulse injection with bidirectional propagation is increased to $60 \%$. The maximum output energies of the twin pulses are $347 \mathrm{~mJ}$ and $351 \mathrm{~mJ}$, and the output energy of a single pulse is only $373 \mathrm{~mJ}$ under the same amplifier operating conditions. The experimental results show that the bidirectional ring amplifier with twin pulses can achieve a higher extraction efficiency of stored energy at a lower operating fluence, and has potential applications in high-power and high-energy laser facilities.
\end{abstract}

Keywords: lasers; pulsed lasers; laser amplifier; ring

\section{Introduction}

Laser amplifiers have been widely used in the fields of engineering applications and scientific research, such as laser material processing ${ }^{[1]}$, plasma physics ${ }^{[2,3]}$, laboratory astrophysics ${ }^{[4,5]}$, inertial confinement fusion $(\mathrm{ICF})^{[6-9]}$ and other important technologies. In the development of highenergy and high-power laser systems, the master oscillator power amplifier (MOPA) was used to realize power amplification with a high beam quality. In general, a laser system with a MOPA scheme is huge, and the extraction efficiency of stored energy is low. More recently, the multi-pass amplifier (MA) has replaced the MOPA, with the advantage of smaller size in the laser system and higher extraction efficiency ${ }^{[10-12]}$. However, the gain and the extraction efficiency of the MA are limited to an extent by the number of passes that the beam can make through the amplifiers. Moreover, the laser system in the MA should operate at a much higher fluence to achieve a higher extraction efficiency

Correspondence to: J. Wang, No. 390 Qinghe Road, Jiading District, Shanghai 201800, China; X. Yuan, No. 1 Shizi Street, Suzhou 215006, China. Email: wajfeng@163.com (J. Wang); xyuan@suda.edu.cn (X. Yuan).

${ }^{\dagger}$ These two authors contributed equally to this work. of stored energy, which results in a challenge for the damage threshold of optical components under high-power laser irradiation.

To increase the extraction efficiency of stored energy and decrease the operating fluence, several kinds of amplifier have been proposed. In 2013, Zhang et al. proposed the bidirectional amplifier (BA) with twin pulses ${ }^{[13]}$. Simulation results showed that the extraction efficiency of stored energy in the amplifier can reach $61 \%$ under the B integral limit. In 2014, Erlandson proposed a patent for a ring amplifier (RA) with a single pulse for compact, multi-pass pulsed laser amplifiers $^{[14]}$. Although this RA was more compact than the MA, it was difficult to extract the stored energy in the amplifiers efficiently and uniformly due to the single-pulse operation. In our previous work, a bidirectional RA with twin pulses (BRA) ${ }^{[15,16]}$ was proposed. Simulation results show that an extraction efficiency of $62.3 \%$ can be achieved at a lower average fluence of $10.3 \mathrm{~J} / \mathrm{cm}^{2}$ and a lower injection energy of $3.9 \mathrm{~mJ}$ under the B integral limit compared to that of the MA.

In this paper, the amplification and the energy flow of the BRA are studied and demonstrated. The injection energies of the twin pulses (pulse-1 and pulse-2) are $0.29 \mathrm{~mJ}$ and $0.32 \mathrm{~mJ}$ with pulse widths of $2.8 \mathrm{~ns}$ (FWHM), respectively. 


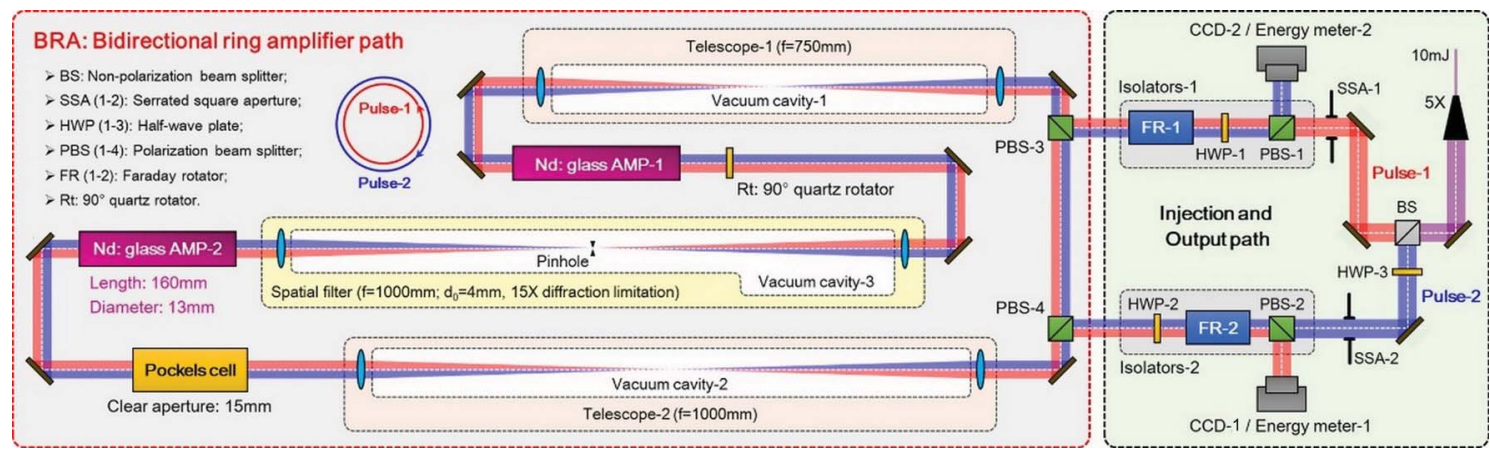

Figure 1. Schematic diagram of the BRA with twin pulses.

The repetition rate of the BRA is $1 \mathrm{~Hz}$. The maximum output energies of the two pulses from the BRA are $347 \mathrm{~mJ}$ and $351 \mathrm{~mJ}$, respectively. Compared to the effective extraction efficiency of $32 \%$ in an RA with a single pulse in the beam area, the maximum effective extraction efficiency of stored energy in the BRA with twin pulses is $60 \%$ under the same operating conditions.

\section{Principle of the BRA with twin pulses}

A BRA with twin pulses was designed and established to verify its output performance. The schematic diagram of the BRA is shown in Figure 1. Two isolators composed of polarization beam splitters (PBS-1 and PBS-2), Faraday rotators (FR-1 and FR-2) and half-wave plates (HWP-2 and HWP-3) are used to ensure the BRA system operates safely and to control pulse injection. The image relay of the BRA is achieved by two telescopes and a spatial filter. The focal length of the lenses in telescope-1 $(750 \mathrm{~mm})$ is different from that of the telescope-2 $(1000 \mathrm{~mm})$ to avoid the two beams colliding at the location of the pinhole in the spatial filter, which is helpful in eliminating air break-down and pinhole closure. Two Nd:glass rods (N3112, developed at the Shanghai Institute of Optics and Fine Mechanics (SIOM)) with diameters of $13 \mathrm{~mm}$ and lengths of $160 \mathrm{~mm}$ are placed at the image relay plane of the spatial filter. The stimulated emission cross-section of $\mathrm{Nd}$ :glass is $3.8 \times 10^{-20} \mathrm{~cm}^{2}$, the saturation fluence is about $4.967 \mathrm{~J} / \mathrm{cm}^{2}$, and the dynamic absorption coefficient is $0.0033 \mathrm{~cm}^{-1}$. The small-signal gain of Nd:glass AMP-1 is about 1.83, and the small-signal gain of Nd:glass AMP-2 is about 2.0. A Pockels cell (PC, clear aperture $15 \mathrm{~mm}$, model 5046E, FastPulse Technology, Inc.) is used to control the polarization of the laser pulses, pulse injection and switch-out. The image area of the CCD (Camyu, Chongqing Camyu Hi-tech Development Co., Ltd.) is $13.6 \mathrm{~mm} \times 13.6 \mathrm{~mm}$, which includes $1024 \times 1024$ active pixels, with the size of each pixel being $13.3 \mu \mathrm{m} \times 13.3 \mu \mathrm{m}$.

The injection laser pulse used in the experiment is from a regenerative amplifier with a center wavelength of $1053 \mathrm{~nm}$, a repetition rate of $1 \mathrm{~Hz}$, and an output energy of about

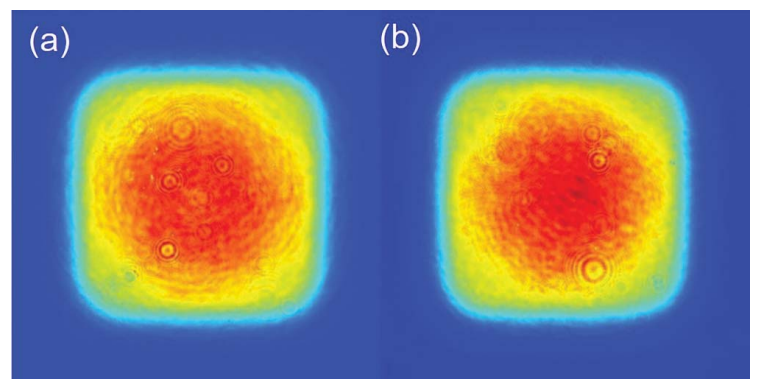

Figure 2. Near-field beam profiles of the injection pulses: (a) pulse-1 and (b) pulse-2.

$10 \mathrm{~mJ}$. The diameter of the laser pulse is expanded from $3 \mathrm{~mm}$ to $15 \mathrm{~mm}$ by a $5 \times$ beam expander, and divided into pulse- 1 and pulse- 2 by a nonpolarizing beam splitter (BS). The near-field profiles of the two injection pulses are shaped with a serrated square aperture (SSA) of size $5 \mathrm{~mm} \times 5 \mathrm{~mm}$ to obtain uniform and collimated incident beams, as shown in Figure 2. After being shaped, pulse-1 passes through the isolators-1, and is injected into the amplifier ring (as shown in the red dashed block in Figure 1) by PBS-3 with the s-polarization in the counterclockwise direction. Pulse-1 passes through telescope- 1 and is amplified by the Nd:glass AMP-1. Telescope-1 relays the image of the beam from SSA-1 to the Nd:glass AMP-1 in order to avoid diffraction effects during beam propagation in the amplifier. Then, pulse-1 passes through the $90^{\circ}$ quartz rotator (Rt) and enters a spatial filter with a cut-off frequency of 15 times the diffraction limit, which relays the beam from the Nd:glass AMP-1 to the Nd:glass AMP-2. After amplification in the Nd:glass AMP-2, pulse-1 is incident on the Pockels cell with zero voltage at the first round (the Pockels cell with high and zero voltages can be considered as operating in on and off states, respectively). Then pulse-1 passes through telescope2 and PBS-4 with the p-polarization after the Pockels cell. Telescope- 2 relays the beam from the Nd:glass AMP-2 to the object plane of telescope-1, and can also relay the image of pulse-2 from SSA-2 to the Nd:glass AMP-2 for pulse-2 injection in the clockwise direction.

The operating state of the Pockels cell is changed to the on state for the second round, and pulse- 1 can be trapped and 


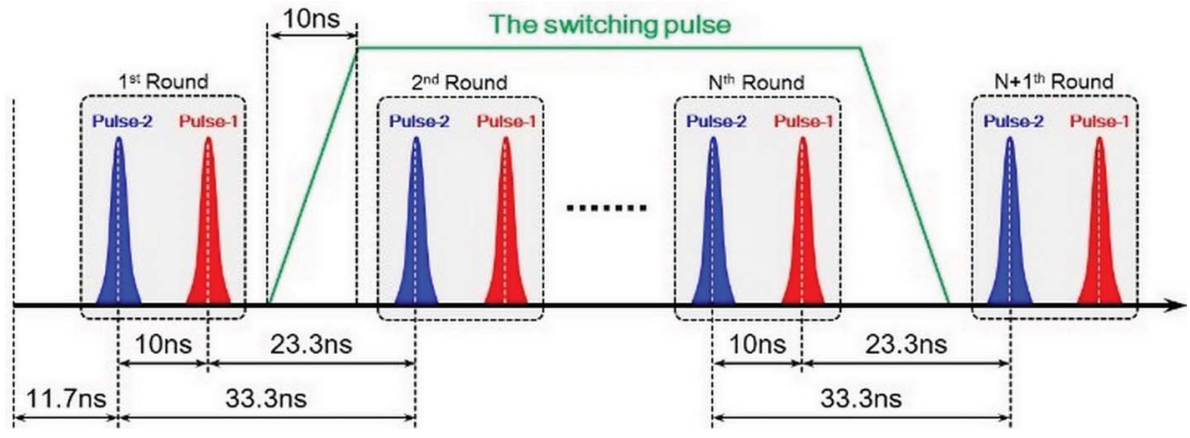

Figure 3. Sequence diagram of the Pockels cell.
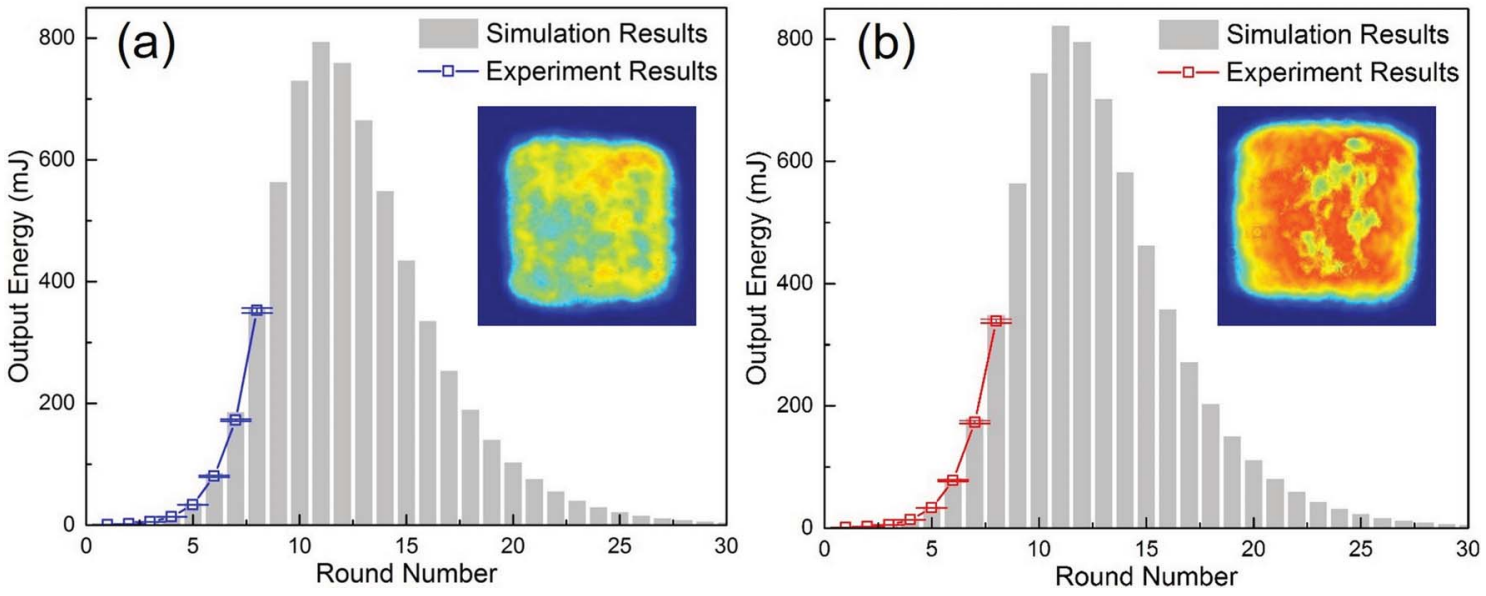

Figure 4. Dependence of the output energy on the number of round trips for single-pulse amplification. (a) Single pulse-1 injected into the RA; (b) single pulse-2 injected into the RA.

amplified in the amplifier ring with the p-polarization. Once pulse-1 passes through a predetermined number of round trips in the amplifier ring, the Pockels cell is set to the off state, and the pulse with the s-polarization can be switched out from PBS-4 at the next round.

The propagation and amplification sequence of pulse- 2 through the RA is similar to that of pulse- 1 , but has a clockwise propagation direction in the amplifier ring. The two pulses are synchronously injected into the amplifier ring, and the states of the Pockels cell are changed between on and off to control the polarization of the pulses. The sequence diagram of the Pockels cell is shown in Figure 3. The red pulse represents pulse-1, the blue pulse represents pulse2 and the green line represents the switching pulse of the Pockels cell. The interval for the two pulses to reach the Pockels cell is about $23.3 \mathrm{~ns}$, which is much longer than the rise and fall time of the switching pulse.

\section{Results and discussions}

The following results are obtained in the experiment. The output performance of single-pulse amplification is shown in Figure 4, and the output performance of twin-pulse amplification is shown in Figure 5. The propagation and amplification in the RA are also simulated based on amplification theory ${ }^{[17,18]}$. The amplifier ring has an approximately $83 \%$ static single-pass transmittance, and the average single-pass effective gain of the two amplifiers is approximately 2.5 .

The dependence of the output energy on the number of round trips in the RA with single-pulse injection is shown in Figure 4, in which the blue line shows the experimental results for pulse-1, the red line shows the experimental results for pulse-2, and the gray bars are simulation results. The experimental results match well with the simulation results. The simulation shows that the maximum output energies of pulse-1 and pulse-2 reach $793 \mathrm{~mJ}$ and $822 \mathrm{~mJ}$ at the 11th round with injection energies of $0.29 \mathrm{~mJ}$ and $0.32 \mathrm{~mJ}$, and the output fluences are $3.1 \mathrm{~J} / \mathrm{cm}^{2}$ and $3.3 \mathrm{~J} / \mathrm{cm}^{2}$, respectively. In the experiment, due to the damage threshold for the optical components, pulse- 1 and pulse- 2 can only propagate for eight rounds in the RA. Therefore, the experimental results for single-pulse injection are that the output energies of pulse- 1 or pulse- 2 are $373 \mathrm{~mJ}$ or $358 \mathrm{~mJ}$ at the 8 th round with injection energies of $0.29 \mathrm{~mJ}$ or $0.32 \mathrm{~mJ}$, and the output fluences are $1.49 \mathrm{~J} / \mathrm{cm}^{2}$ or $1.43 \mathrm{~J} / \mathrm{cm}^{2}$, respectively. 

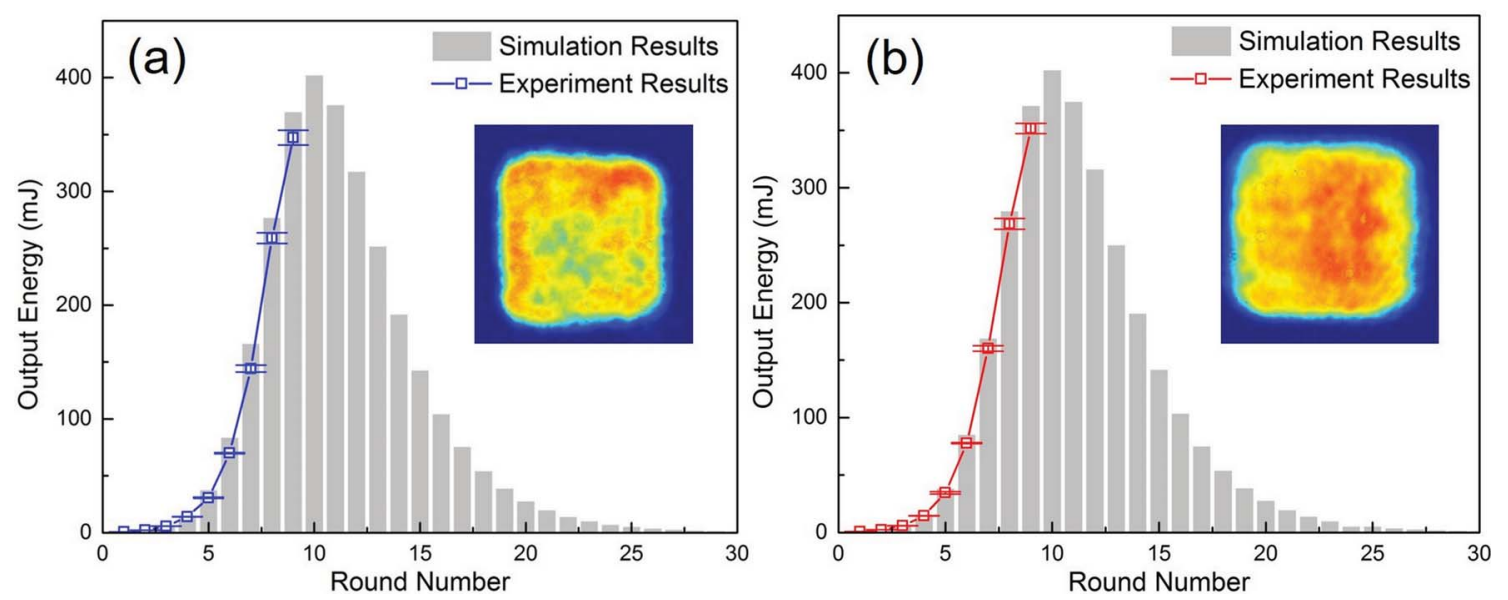

Figure 5. Dependence of output energy on the number of round trips for the BRA with twin pulses. (a) Output energy of pulse-1; (b) output energy of pulse-2.

Also, the effective extraction efficiencies of stored energy for pulse- 1 and pulse- 2 are approximately $32 \%$ or $30 \%$ in the beam area, respectively, where the effective extraction efficiency is defined as the ratio of the output energy to the stored energy in the beam area.

The dependence of output energy on the number of round trips for the BRA with twin-pulse injection is shown in Figure 5. The experimental results are shown as the blue line for pulse- 1 and red line for pulse-2, and the simulation results are shown as the gray bars. The output energies of pulse- 1 and pulse- 2 are $259 \mathrm{~mJ}$ and $268 \mathrm{~mJ}$ at the 8th round with the same injection energy as that for the singlepulse injection, and the output fluences are $1.03 \mathrm{~J} / \mathrm{cm}^{2}$ and $1.07 \mathrm{~J} / \mathrm{cm}^{2}$, respectively. The effective extraction efficiency of stored energy is approximately $45 \%$ in the beam area. The extraction efficiency of stored energy at the 8th round of the BRA with twin pulses is increased by $41 \%$ compared with that of the RA with a single pulse under the same conditions. Therefore, the output fluences of the BRA with twin pulses are much lower than those of the RA with a single pulse, which can greatly increase the reliability and reduce the cost of the laser system.

Additionally, the maximum output energies of pulse- 1 and pulse- 2 are $347 \mathrm{~mJ}$ and $352 \mathrm{~mJ}$ achieved at the 9th round, and the output fluences are $1.39 \mathrm{~J} / \mathrm{cm}^{2}$ and $1.41 \mathrm{~J} / \mathrm{cm}^{2}$, respectively. The effective extraction efficiency of stored energy is approximately $60 \%$ in the beam area, which is much higher than that of the RA with single-pulse injection under the same operating fluence. The simulation results show that the maximum output energies of both pulse- 1 and pulse- 2 reach $402 \mathrm{~mJ}$ at the 10th round, as shown in Figure 5. To ensure safe operation of the BRA system, a high-fluence operation experiment was not carried out. We intend to improve our experimental setup with high-damage-threshold optical components, a high-quality Pockels cell and a serrated square aperture, and carry out a high-quality, high-fluence experiment to optimize the characteristics of the BRA.

\section{Conclusions}

In summary, the output performances of a BRA with twin pulses have been studied and demonstrated. The experimental results show that the effective extraction efficiency for the BRA with twin pulses can be up to $60 \%$, which is much higher than that of the RA with a single pulse of $32 \%$. Moreover, the operating fluence of the BRA with twin pulses is lower than that of the RA with a single pulse, which is important for reliable operation in high-power laser systems and has potential applications in high-power, high-energy lasers. The BRA with twin pulses discussed in this paper operates with a low repetition rate and a low fluence due to the limited experimental conditions, but we will extend our work to high-repetition-rate, high-fluence operation in the future.

\section{Acknowledgements}

This work is supported by Key R\&D Program of China (2016YFF0100903); National Natural Science Foundation of China (NSFC) (61775153, 61705153, 11504255, 61705242); Natural Science Foundation of Jiangsu Province (BK20141232); Priority Academic Program Development of Jiangsu Higher Education Institutions (PAPD); Shanghai Science and Technology Foundation for Young Scholars (17YF1429600).

The authors thank the Shanghai Institute of Optics and Fine Mechanics for the use of their equipment.

\section{References}

1. W. M. Steen and J. Mazumder, Laser Material Processing (Springer, London, 2010).

2. E. Esarey, C. B. Schroeder, and W. P. Leemans, Rev. Mod. Phys. 81, 1229 (2009). 
3. E. I. Moses, R. N. Boyd, B. A. Remington, C. J. Keane, and R. Al-Ayat, Phys. Plasmas 16, 041106 (2009).

4. C. Spindloe, D. Wyatt, S. Astbury, G. F. Swadling, T. Clayson, C. Stehlé, J. M. Foster, E. Gumbrell, R. Charles, C. N. Danson, P. Brummitt, and F. Suzuki-Vidal, High Power Laser Sci. Eng. 3, e22 (2017).

5. F. Wang, X. Pei, B. Han, H. Wei, D. Yuan, G. Liang, G. Zhao, J. Zhong, Z. Zhang, B. Zhu, Y. Li, F. Li, Y. Li, S. Zeng, S. Zou, and J. Zhang, High Power Laser Sci. Eng. 4, e27 (2016).

6. J. T. Hunt and D. R. Speck, Opt. Eng. 28, 461 (1989).

7. C. Bibeau, D. R. Speck, R. B. Ehrlich, C. W. Laumann, D. T. Kyrazis, M. A. Henesian, J. K. Lawson, M. D. Perry, P. J. Wegner, and T. L. Weiland, Appl. Opt. 31, 5799 (1992).

8. W. W. Simmons and R. O. Godwin, Nucl. Tech. Fusion 4, 8 (1983).

9. G. H. Miller, E. I. Moses, and C. R. Wuest, Opt. Eng. 43, 2841 (2004).

10. W. J. Hogan, E. I. Moses, B. E. Warner, M. S. Sorem, and J. M. Soures, Nucl. Fusion 41, 567 (2001).
11. B. Canaud, F. Garaude, C. Clique, N. Lecler, A. Masson, R. Quach, and J. Van der Vliet, Nucl. Fusion 47, 1652 (2007).

12. W. Zheng, X. Wei, Q. Zhu, F. Jing, D. Hu, J. Su, K. Zheng, X. Yuan, H. Zhou, W. Dai, W. Zhou, F. Wang, D. Xu, X. Xie, B. Feng, Z. Peng, L. Guo, Y. Chen, X. Zhang, L. Liu, D. Lin, Z. Dang, Y. Xiang, and X. Deng, High Power Laser Sci. Eng. 4, e21 (2016).

13. X. Zhang, X. Wei, M. Li, Q. Yuan, W. Wang, and F. Li, Laser Phys. Lett. 10, 115803 (2013).

14. A. C. Erlandson, Method and system for compact, multi-pass pulsed laser amplifier. U.S. Patent 8,896,913 (November 25, 2014).

15. T. Yu, F. Gao, X. Zhang, B. Xiong, and X. Yuan, Opt. Express 26, 15300 (2018).

16. X. Yuan, T. Yu, F. Gao, and X. Zhang, High-efficiency highpower ring laser amplifier. U.S. Patent 9,991,664 (June 5, 2018).

17. L. M. Frantz and J. S. Nodvik, J. Appl. Phys. 34, 2346 (1963).

18. W. H. Lowdermilk and J. E. Murray, J. Appl. Phys. 51, 2436 (1980). 